\title{
On the existence for diffeo-integral inclusion of Sobolev-type of fractional order with applications
}

\author{
Rabha W. Ibrahim ${ }^{1}$ \\ (Received 25 March 2008; revised 9 July 2010)
}

\begin{abstract}
By using a suitable fixed point theorems, we study the existence of solutions for fractional diffeo-integral inclusion of Sobolev-type. The study arises in the case when the set-valued function has convex and non-convex values.
\end{abstract}

\section{Contents}

1 Introduction

E2

2 Preliminaries

E4

3 Single-valued problem

E8

4 Set-valued problem

E11

http://anziamj. austms.org.au/ojs/index.php/ANZIAMJ/article/view/1161 gives this article, (c) Austral. Mathematical Soc. 2010. Published July 29, 2010. ISSN 1446-8735. (Print two pages per sheet of paper.) 


\section{Introduction}

Many investigators have proposed the use of fractional time derivatives on a purely mathematical or heuristic basis $[1,2,3]$. From the perspective of theoretical physics this proposal touches upon fundamental principles such as locality, irreversibility and invariance under time translations because fractional derivatives are non-local operators that are not invariant under time reversal [4]. Moreover, the fractional time derivatives with orders between 0 and 1 may generally appear as macroscopic time evolution [4]. There are several kinds of fractional derivatives [5, 6, 7, 8, 9]. El-Sayed and A. G. Ibrahim gave the concept of the definite integral of fractional order for setvalued function $[10,11,12]$. We all know that the existence of the solution for nonlinear (integral, differential and diffeo-integral) inclusion is very important. Many interesting extensions are used in many applications; for example in control systems, fuzzy systems, and dynamic systems $[13,14,15,16]$. We continue the study of this kind of problem by considering the following inclusion

$$
D^{\alpha} u(t) \in F\left(t, u(t), I^{\beta} k(t, u(t))\right), \quad 0<\alpha \leqslant 1, \quad \beta>0, \quad t \in J:=[0, T],
$$

subject to the initial condition $\mathfrak{u}(0)=0$ with $\alpha+\beta \in(0,1]$, where $F$ : $J \times \mathbb{R} \times \mathbb{R} \rightarrow \mathcal{P}(\mathbb{R})$ is compact set-valued function with nonempty values in $\mathbb{R}$, and $\mathcal{P}(\mathbb{R})$ is the family of all nonempty subsets of $\mathbb{R}$. Let $I^{\alpha} F(t, u(t))$ be the definite integral for the set-valued function $F$ of order $\alpha$ :

$$
I^{\alpha} F(t, u(t))=\left\{\frac{1}{\Gamma(\alpha)} \int_{0}^{t}(t-\tau)^{\alpha-1} f(\tau, u(\tau)) d \tau: f(t, u) \in S_{F}(u)\right\}
$$


where

$$
S_{F}(u)=\left\{f \in L^{1}(J, \mathbb{R}): f(t) \in F(t, u(t)), \quad \text { almost everywhere } t \in J\right\}
$$

denotes the set of selections of $F$.

This article consists of the following sections. Section 2 deals with some basic definitions and preliminary facts from set-valued analysis to be used. Section 3 establishes the existence of solution for the single-valued problem

$$
\begin{aligned}
& D^{\alpha} u(t)=f\left(t, u(t), I^{\beta} k(t, u(t))\right), \\
& 0<\alpha \leqslant 1, \quad \beta>0, \quad t \in J, \quad f \in F\left(t, u(t), I^{\beta} k(t, u(t))\right),
\end{aligned}
$$

subject to the initial condition $u(0)=0$ using the Schauder fixed point theorem [17, 18, 19], and uniqueness of solutions using the Banach fixed point theorem [20]. Section 4 studies the existence of solutions for the set-valued problem (1) when F has convex as well as non-convex values via the singlevalued problem as well as fixed point theorems of the set-valued function. In the first case (convex) a generalization of the Leray-Schauder principle for set-valued functions is used [21]. A fixed point theorem for contraction set-valued functions due to Covitz and Nadler [22] is applied in the second one (non-convex). We also illustrate our results with an example.

As an application of the fractional diffeo-integral inclusions and equations covers widely known classical fields and topics. Viscoelasticity appears to be the field of the most extensive applications of diffeo-integral inclusions and equations, and perhaps the only one. In this case the mathematical modeling of viscoelastic materials is quite natural. For example, the wellknown relationship between stress and strain for solids is

$$
\begin{aligned}
\sigma(t) & =E D^{\alpha} \epsilon(t), \quad 0 \leqslant \alpha<1, \\
\text { with } \epsilon(t) & =\frac{1}{k} I^{\beta} g(t), \quad \beta>0,
\end{aligned}
$$

where $E$ and $k$ are material constants and $g$ is a continuous function on $\mathrm{J}=[0, \mathrm{~T}]$, which represents as an ideal solid material. The main reasons for 
the theoretical development of fractional order (non-integer order) models are mainly the wide use of polymers in various fields of engineering. Moreover, the fractional laws of deformation find uses in solving practical problems of viscoelasticity [6].

Also the fractional diffeo-integral inclusion of Sobolev-type appears in the theory of control of dynamical systems, when the controlled system or/and the controller is described by a fractional diffeo-integral inclusion of Sobolev-type and for specific case by diffeo-integral equation of Sobolev-type. Furthermore, the mathematical modeling and simulations of systems and processes are based on the description of their properties in term of fractional diffeointegral inclusion of Sobolev-type. These new models are more adequate than previously used integer order models. Inclusions and equations involving both fractional operators (differential and integral) play the role of 'reality' and 'model'. For example, we set a control fractional differential equation as follows: let $\mathrm{K}_{\mathrm{c}}\left(\mathbb{R}^{\mathrm{n}}\right)$ denote the collection of all nonempty compact convex subsets of $\mathbb{R}^{n}$, then the fractional derivative of order $\alpha \in(0,1]$ is

$$
\mathrm{D}^{\alpha} \mathrm{X}(\mathrm{t})=\mathrm{F}\left(\mathrm{t}, \mathrm{X}(\mathrm{t}), \mathrm{I}^{\beta} \mathrm{U}(\mathrm{t})\right), \quad 0<\alpha \leqslant 1,
$$

$$
\begin{gathered}
\text { where } X(0)=0, \quad X(t) \in K_{c}\left(\mathbb{R}^{n}\right), \quad I^{\beta} U(t) \in K_{c}\left(\mathbb{R}^{m}\right), \quad t \in J:=[0, T], \\
F\left(t, X(t), I^{\beta} U(t)\right): J \times K_{c}\left(\mathbb{R}^{n}\right) \times K_{c}\left(\mathbb{R}^{m}\right) \longrightarrow K_{c}\left(\mathbb{R}^{n}\right) .
\end{gathered}
$$

\section{Preliminaries}

The following preliminary facts from set-valued analysis are used throughout this article [23, 24, 25, 26, 27, 28, 29].

$\mathcal{B}:=\mathrm{C}\left[\mathrm{J}, \mathbb{R}^{n}\right]$ is a Banach space of all continuous functions from $\mathrm{J}$ into $\mathbb{R}^{n}$ with the norm

$$
\|u\|_{\infty}=\sup \{|u(t)|: t \in J\}, \quad \text { for all } u \in \mathcal{B} .
$$


$\mathcal{L}:=\mathrm{L}^{1}\left[\mathrm{~J}, \mathbb{R}^{\mathrm{n}}\right]$ denotes the Banach space of measurable functions $\mathrm{u}: \mathrm{J} \rightarrow \mathbb{R}^{\mathrm{n}}$ which are Lebesgue integrable normed by

$$
\|u\|_{L^{1}}=\int_{0}^{T}|u(t)| d t, \quad \text { for all } u \in \mathcal{L}
$$

Let $(X,||$.$) be a Banach space,$

- $\mathcal{P}_{\mathrm{cl}}(\mathrm{X})=\{\mathrm{Y} \in \mathcal{P}(\mathrm{X}): \mathrm{Y}$ is closed $\}$,

- $\mathcal{P}_{\mathrm{b}}(\mathrm{X})=\{\mathrm{Y} \in \mathcal{P}(\mathrm{X}): \mathrm{Y}$ is bounded $\}$,

- $\mathcal{P}_{\mathrm{cp}}(\mathrm{X})=\{\mathrm{Y} \in \mathcal{P}(\mathrm{X}): \mathrm{Y}$ is compact $\}$,

- $\mathcal{P}_{\mathfrak{c}}(\mathrm{X})=\{\mathrm{Y} \in \mathcal{P}(\mathrm{X}): \mathrm{Y}$ is convex $\}$,

- $\mathcal{P}_{\mathrm{cl}, \mathrm{c}}(\mathrm{X})=\{\mathrm{Y} \in \mathcal{P}(\mathrm{X}): \mathrm{Y}$ is closed and convex $\}$,

- $\mathcal{P}_{\mathfrak{c p}, \mathrm{c}}(\mathrm{X})=\{\mathrm{Y} \in \mathcal{P}(\mathrm{X}): \mathrm{Y}$ is compact and convex $\}$.

In addition,

- a set-valued function $F: X \rightarrow \mathcal{P}(X)$ is called convex (closed) valued if $\mathrm{F}(\mathrm{x})$ is convex (closed) for all $\mathrm{x} \in \mathrm{X}$.

- $F$ is called bounded valued on bounded set $B$ if $F(B)=\bigcup_{x \in B} F(x)$ is bounded in $X$ for all $B \in \mathcal{P}_{\mathfrak{b}}(X)$; that is, $\sup _{x \in B}\{\sup \{|\mathfrak{u}|: \mathfrak{u} \in \mathrm{F}(\mathrm{x})\}\}<$ $\infty$.

- $F$ is called upper semi-continuous on $X$ if for each $x_{0} \in X$ the set $F\left(x_{0}\right)$ is nonempty closed subset of $X$ and if for each open set $N$ of $X$ containing $F\left(x_{0}\right)$, there exist an open neighbourhood $N_{0}$ of $x_{0}$ such that $\mathrm{F}\left(\mathrm{N}_{0}\right) \subseteq \mathrm{N}$. In other words, $\mathrm{F}$ is upper semi-continuous if the set $F^{-1}(A)=\{x \in X: F(x) \subset A\}$ is open in $X$ for every open set $A$ in $X$.

- $F$ is called lower semi-continuous on $X$ if $A$ is any open subset of $X$ then $F^{-1}(A)=\{x \in X: F(x) \cap A \neq \emptyset\}$ is open in $X$. 
- $F$ is called continuous if it is lower as well as upper semi-continuous on $X$.

- $F$ is called compact if for every $M$ bounded subset of $X, F(M)$ is relatively compact.

- Finally, F is called completely continuous if it is upper semi-continuous and compact on $\mathrm{X}$.

The following definitions are used in subsequent sections.

Definition 1 A mapping $\mathrm{p}: \mathrm{J} \times \mathbb{R}^{n} \times \mathbb{R}^{n} \rightarrow \mathbb{R}^{n}$ is Carathéodory if

1. $\mathrm{t} \rightarrow \mathrm{p}(\mathrm{t}, \mathrm{u}, \boldsymbol{v})$ is measurable for each $(\mathfrak{u}, \boldsymbol{v}) \in \mathbb{R}^{\mathrm{n}} \times \mathbb{R}^{\mathrm{n}}$,

2. $(\mathfrak{u}, v) \rightarrow p(t, u, v)$ is continuous almost everywhere for $\mathrm{t} \in \mathrm{J}$.

Definition 2 A set-valued function $\mathrm{F}: \mathrm{J} \rightarrow \mathcal{P}\left(\mathbb{R}^{n}\right)$ is measurable if for any $x \in \mathrm{X}$, the function $\mathrm{t} \mapsto \mathrm{d}(\mathrm{x}, \mathrm{F}(\mathrm{t}))=\inf \{|\mathrm{x}-\mathrm{u}|: \mathrm{u} \in \mathrm{F}(\mathrm{t})\}$ is measurable.

Definition 3 A set-valued function $\mathrm{F}: \mathrm{J} \times \mathbb{R}^{n} \times \mathbb{R}^{n} \rightarrow \mathcal{P}\left(\mathbb{R}^{n}\right)$ is called Carathéodory if

1. $\mathrm{t} \mapsto \mathrm{F}(\mathrm{t}, \mathrm{x}, \mathrm{y})$ is measurable for each $(\mathrm{x}, \mathrm{y}) \in \mathbb{R}^{\mathrm{n}} \times \mathbb{R}^{\mathrm{n}}$, and

2. $(\mathrm{x}, \mathrm{y}) \mapsto \mathrm{F}(\mathrm{t}, \mathrm{x}, \mathrm{y})$ is upper semi-continuous for almost $\mathrm{t} \in \mathrm{J}$.

Let $A, B \in \mathcal{P}_{c l, b}(X)$ and let $a \in A$. Then by

$\mathrm{D}(\mathrm{a}, \mathrm{B})=\inf \{\|\mathrm{a}-\mathrm{b}\|: \mathrm{b} \in \mathrm{B}\} \quad$ and $\quad \rho(\mathrm{A}, \mathrm{B})=\sup \{\mathrm{D}(\mathrm{a}, \mathrm{B}): \mathrm{a} \in \mathrm{A}\}$,

the Hausdorff metric $\mathrm{H}: \mathcal{P}_{\mathrm{cl}, \mathrm{b}}(\mathrm{X}) \times \mathcal{P}_{\mathrm{cl}, \mathrm{b}}(\mathrm{X}) \rightarrow \mathbb{R}^{+}$is defined by

$$
H(A, B)=\max \{\rho(A, B), \rho(B, A)\}
$$

such that

$$
\mathrm{H}(\mathrm{O}, \mathrm{C})=\sup \left\{\|\mathrm{c}\|: \mathrm{c} \in \mathrm{C}, \mathrm{C} \in \mathcal{P}_{\mathrm{cl}, \mathrm{b}}(\mathrm{X})\right\} .
$$

Moreover, $\left(\mathcal{P}_{\mathrm{cl}, \mathrm{b}}(\mathrm{X}), \mathrm{H}\right)$ is a complete metric space [30].

Definition 4 A set-valued function $\mathrm{F}: \mathbb{R} \rightarrow \mathcal{P}_{\text {cl }}(\mathbb{R})$ is called 
1. $\gamma$-Lipschitz if and only if there exists $\gamma>0$ such that

$$
\mathrm{H}(\mathrm{F}(\mathrm{x}), \mathrm{F}(\mathrm{y})) \leqslant \gamma\|\mathrm{x}-\mathrm{y}\|, \quad \text { for each } \mathrm{x}, \mathrm{y} \in \mathrm{X} .
$$

The constant $\gamma$ is called a Lipschitz constant.

2. a contraction if and only if it is $\gamma$-Lipschitz with $\gamma<1$.

Definition 5 A set-valued function $\mathrm{F}: \mathrm{J} \times \mathbb{R} \rightarrow \mathcal{P}_{\text {cl }}(\mathbb{R})$ is called

1. $\gamma(\mathrm{t})$-Lipschitz if and only if there exists $\gamma \in \mathrm{L}^{1}\left(\mathrm{~J}, \mathbb{R}^{+}\right)$such that

$$
\mathrm{H}(\mathrm{F}(\mathrm{t}, \mathrm{x}), \mathrm{F}(\mathrm{t}, \mathrm{y})) \leqslant \gamma(\mathrm{t})\|\mathrm{x}-\mathrm{y}\|, \quad \text { for each } \mathrm{x}, \mathrm{y} \in \mathrm{X} .
$$

2. a contraction if and only if it is $\gamma(\mathrm{t})$-Lipschitz with $\|\gamma\|_{\mathrm{L}^{1}}<1$.

Definition 6 A measurable set-valued function $\mathrm{F}: \mathrm{J} \rightarrow \mathcal{P}_{\mathrm{cp}}(\mathbb{R})$ is integrably bounded if there exists a function $\mathrm{h} \in \mathrm{L}^{1}\left(\mathrm{~J}, \mathbb{R}^{+}\right)$such that $\|v\| \leqslant \mathrm{h}(\mathrm{t})$ almost everywhere $\mathrm{t} \in \mathrm{J}$ for all $\boldsymbol{v} \in \mathrm{F}(\mathrm{t})$.

Definition 7 The fractional (arbitrary) order integral of the function $f$ of order $\alpha>1$ is defined by [5, 6, 7, 8,9]

$$
I_{a}^{\alpha} f(t)=\int_{a}^{t} \frac{(t-\tau)^{\alpha-1}}{\Gamma(\alpha)} f(\tau) d \tau
$$

When $\mathrm{a}=0$, we write $\mathrm{I}_{\mathrm{a}}^{\alpha} \mathrm{f}(\mathrm{t})=\mathrm{f}(\mathrm{t}) * \phi_{\alpha}(\mathrm{t})$, where $(*)$ denoted the convolution product [5], $\phi_{\alpha}(\mathrm{t})=\mathrm{t}^{\alpha-1} / \Gamma(\alpha), \mathrm{t}>0$, and $\phi_{\alpha}(\mathrm{t})=0, \mathrm{t} \leqslant 0$, and $\phi_{\alpha} \rightarrow \delta(\mathrm{t})$ as $\alpha \rightarrow 0$ where $\delta(\mathrm{t})$ is the delta function.

Definition 8 The fractional (arbitrary) order derivative of the function $f$ of order $\alpha>1$ is defined by [5, 6, 7, 8, 9]

$$
D_{a}^{\alpha} f(t)=\frac{d}{d t} \int_{a}^{t} \frac{(t-\tau)^{-\alpha}}{\Gamma(1-\alpha)} f(\tau) d \tau=\frac{d}{d t} I_{a}^{1-\alpha} f(t) .
$$

The following remarks and lemmas are used in remaining sections. 
Remark 9 [31] Let $M \subset X$. If the graph of $F: M \rightarrow \mathcal{P}(X)$ is closed and $F(M)$ is relatively compact, then $F$ is upper semi-continuous on $M$.

Remark 10 [25] It is known that if $\mathrm{F}: \mathrm{J} \rightarrow \mathcal{P}_{\mathrm{cp}}(\mathbb{R})$ is a measurable set-valued operator, then the set $S_{\mathrm{F}}^{1}$ of all Lebesgue integrable selections of $F$ is closed and nonempty.

Remark $11[5,6]$ The fractional order derivative and integral for a continuous function $f$ have the relation

$$
I_{a}^{\alpha}\left(D_{a}^{\alpha} f(t)\right)=D_{a}^{\alpha}\left(I_{a}^{\alpha} f(t)\right)=f(t) .
$$

Lemma 12 [21] Consider the operator $\mathrm{F}: \mathrm{X} \rightarrow \mathcal{P}(\mathrm{X})$. Suppose that

1. for all $\mathrm{x} \in \mathrm{X}, \mathrm{F}(\mathrm{x})$ is a closed, convex and compact set-valued operator,

2. $\mathrm{F}$ is upper semi-continuous on $\mathrm{X}$.

Then either the set $A:=\{x \in X:$ there exists $\lambda \in(0,1), x \in \lambda F(x)\}$ is unbounded or there exists a fixed point $\mathrm{x} \in \mathrm{X}$ such that $\mathrm{x} \in \mathrm{F}(\mathrm{x})$.

Lemma 13 (Covitz-Nadler) [22] Let (X, d) be a complete metric space. If $\mathrm{G}: \mathrm{X} \rightarrow \mathcal{P}_{\mathrm{cl}}(\mathrm{X})$ is a contraction, then $\mathrm{G}$ has a fixed point.

\section{Single-valued problem}

Let $|\cdot|$ be the norm on $\mathbb{R}^{n}$. Define a Banach space $\mathcal{B}:=\mathrm{C}\left[\mathrm{J}, \mathbb{R}^{\mathrm{n}}\right]$. We establish the existence and uniqueness solution for the fractional diffeo-integral equation (2). For this purpose we impose the following assumptions.

Assumption $\mathbf{H 1}$ The function $\mathrm{f}$ is Carathéodory.

Assumption H2 Assume that

$$
|f(t, u, v)| \leqslant \rho(t)\left(\|u\|_{\infty}+\|v\|_{\infty}\right),
$$

where $\|\mathrm{u}\|_{\infty}:=\sup _{\mathrm{t} \in \mathrm{J}}\{|\mathrm{u}(\mathrm{t})|\}$ and $\rho \in \mathrm{L}^{1}\left(\mathrm{~J}, \mathbb{R}_{+}\right)$. 
Assumption H3 There exists a positive function $\mathrm{p} \in \mathrm{C}\left(\mathrm{J}, \mathbb{R}_{+}\right)$such that $|\mathrm{k}(\mathrm{t}, \mathrm{u})| \leqslant \mathrm{p}(\mathrm{t})\left(1+\|\mathrm{u}\|_{\infty}\right)$ implies that

$$
I^{\beta}|k(t, u)| \leqslant \frac{\|p\|_{\infty} T^{\beta}}{\Gamma(\beta+1)}\left(1+\|u\|_{\infty}\right), \quad t \in[0, T] .
$$

Remark 14 In view of Remark 11, we easily find that equation (2) is equivalent to the integral equation of the form

$$
u(t)=I^{\alpha} f\left(t, u(t), I^{\beta} k(t, u(t))\right),
$$

that is, the existence of solution of (2) is equivalent to the existence of solution of (3).

Theorem 15 Let H1-H3 hold. Then equation (2) has a solution $\mathbf{u}(\mathbf{t})$ on $\mathbf{J}$.

Proof: Define an operator $\mathrm{P}: \mathcal{B} \rightarrow \mathcal{B}$ as

$$
(P u)(t):=I^{\alpha} f\left(t, u(t), I^{\beta} k(t, u(t))\right) .
$$

In order to show that (1) has a solution we have to show that the operator (4) has a fixed point.

$$
\begin{aligned}
|(P u)(t)| & =\left|I^{\alpha} f\left(t, u(t), I^{\beta} k(t, u(t))\right)\right| \\
& \leqslant I^{\alpha}\left|f\left(t, u(t), I^{\beta} k(t, u(t))\right)\right| \\
& \leqslant \frac{T^{\alpha}}{\Gamma(\alpha+1)}\|\rho\|_{L^{1}}\left(\|u\|_{\infty}+\left|I^{\beta} k(t, u(t))\right|\right) \\
& \leqslant \frac{T^{\alpha}}{\Gamma(\alpha+1)}\|\rho\|_{L^{1}}\left(\|u\|_{\infty}+I^{\beta}|k(t, u(t))|\right) \\
& \leqslant \frac{T^{\alpha}}{\Gamma(\alpha+1)}\|\rho\|_{L^{1}}\left[\|u\|_{\infty}+\frac{\|p\|_{\infty} T^{\beta}}{\Gamma(\beta+1)}\left(1+\|u\|_{\infty}\right)\right]
\end{aligned}
$$


Thus we obtain that $\|\mathrm{P}\| \leqslant \mathrm{r}, \mathrm{r}>0$, where

$$
r:=\frac{\left.\|\rho\|_{\mathrm{L}^{1}} \mathrm{~T}^{\alpha+\beta}\|p\|_{\infty}\right]}{\Gamma(\beta+1) \Gamma(\alpha+1)}\left[1-\frac{\|\rho\|_{\mathrm{L}^{1}} \mathrm{~T}^{\alpha}}{\Gamma(\alpha+1)}\left(1+\frac{\mathrm{T}^{\beta}\|\mathrm{p}\|_{\infty}}{\Gamma(\beta+1)}\right)\right]^{-1} .
$$

Then $P$ maps $B_{r}$ into itself: $P$ maps the convex closure of $P\left[B_{r}\right]$ into itself for sufficiently small $T>0$. Since $f$ is bounded on $B_{r}, P\left[B_{r}\right]$ is equicontinuous (Arzela-Ascoli): for $0 \leqslant t_{1} \leqslant t_{2} \leqslant T$ such that $\left|t_{2}-t_{1}\right| \leqslant \delta, \delta>0$ and $u \in B[0, r]$,

$$
\begin{aligned}
\left|P u\left(t_{1}\right)-P u\left(t_{2}\right)\right| & \leqslant\left[\frac{\|f\|}{\Gamma(\alpha+1)}\right]\left|\left(t_{1}^{\alpha}-t_{2}^{\alpha}+2\left(t_{1}-t_{2}\right)^{\alpha}\right)\right| \\
& \leqslant\left[\frac{2\|f\|}{\Gamma(\alpha+1)}\right]\left|\left(t_{2}-t_{1}\right)^{\alpha}\right| \leqslant\left[\frac{2\|f\|}{\Gamma(\alpha+1)}\right]\left|\left(t_{2}-t_{1}\right)\right|^{\alpha} \\
& \leqslant \frac{2 \delta^{\alpha}\|f\|}{\Gamma(\alpha+1)} .
\end{aligned}
$$

Hence the Schauder fixed point theorem shows that $\mathrm{P}$ has a fixed point $u \in \mathcal{B}$ such that $\mathrm{Pu}(\mathrm{t})=\mathfrak{u}(\mathrm{t})$, which corresponds to the solution of (2).

Now we discuss the uniqueness of solution for the single-valued problem (2). For this purpose let us impose the following assumptions

Assumption $\mathbf{H} 4$ The function $\mathbf{f}$ satisfies that there exist a function $\mathrm{L}(\mathrm{t}) \in$ $\mathrm{L}^{1}\left(\mathrm{~J}, \mathbb{R}^{+}\right)$such that for each $\mathfrak{u}_{1}, v_{1}$ and $\mathfrak{u}_{2}, v_{2} \in \mathcal{B}$, we have

$$
\left|f\left(t, u_{1}(t), v_{1}(t)\right)-f\left(t, u_{2}(t), v_{2}(t)\right)\right| \leqslant L(t)\left[\left\|u_{1}-u_{2}\right\|_{\infty}+\left\|v_{1}-v_{2}\right\|_{\infty}\right] .
$$

Assumption $\mathbf{H 5}$ The function $\mathrm{k}$ satisfies that there exist a function $\ell(\mathrm{t}) \in$ $\mathrm{L}^{1}\left(\mathrm{~J}, \mathbb{R}^{+}\right)$such that for each $\mathfrak{u}_{1}, \mathfrak{u}_{2} \in \mathcal{B}$ we have

$$
\left|k\left(t, u_{1}(t)\right)-k\left(t, u_{2}(t)\right)\right| \leqslant \ell(t)\left\|u_{1}-u_{2}\right\|_{\infty} .
$$

Theorem 16 Let H4 and H5 hold. If

$$
\frac{\|\mathrm{L}\|_{L^{1}} \mathrm{~T}^{\alpha}\left[\Gamma(\beta+1)+\|\ell\|_{\mathrm{L}^{1}} \mathrm{~T}^{\beta}\right]}{\Gamma(\beta+1) \Gamma(\alpha+1)}<1,
$$

then (2) admits a unique solution $\mathfrak{u}(\mathrm{t})$ on $\mathrm{J}$. 
Proof: Recalling the operator P defined in equation (4), we only need to show that $\mathrm{P}$ is a contraction mapping; that is, $\mathrm{P}$ has a unique fixed point which corresponds to the unique solution of the equation (2). Let $\mathfrak{u}_{1}, \mathfrak{u}_{2} \in \mathcal{B}$ then for all $t \in J$, we obtain that

$$
\begin{aligned}
& \left|\left(P u_{1}\right)(t)-\left(P u_{2}\right)(t)\right| \\
& \leqslant I^{\alpha}\left|f\left(t, u_{1}(t), I^{\beta} k\left(t, u_{1}(t)\right)\right)-f\left(t, u_{2}(t), I^{\beta} k\left(t, u_{2}(t)\right)\right)\right| \\
& \leqslant \frac{T^{\alpha}}{\Gamma(\alpha+1)}\|L\|_{L^{1}}\left[\left\|u_{1}-u_{2}\right\|_{\infty}+\left\|I^{\beta} k\left(t, u_{1}(t)\right)-I^{\beta} k\left(t, u_{2}(t)\right)\right\|\right] \\
& \leqslant \frac{T^{\alpha}}{\Gamma(\alpha+1)}\|L\|_{L^{1}}\left[\left\|u_{1}-u_{2}\right\|_{\infty}+I^{\beta}\left\|k\left(t, u_{1}(t)\right)-k\left(t, u_{2}(t)\right)\right\|\right] \\
& \leqslant \frac{T^{\alpha}}{\Gamma(\alpha+1)}\|L\|_{L^{1}}\left[\left\|u_{1}-u_{2}\right\|_{\infty}+\frac{T^{\beta}}{\Gamma(\beta+1)}\|\ell\|_{L^{1}}\left\|u_{1}-u_{2}\right\|_{\infty}\right] \\
& \leqslant \frac{\|L\|_{L^{1}} T^{\alpha}}{\Gamma(\alpha+1)}\left[\left\|u_{1}-u_{2}\right\|_{\infty}+\frac{\|\ell\|_{L^{1}} T^{\beta}}{\Gamma(\beta+1)}\left\|u_{1}-u_{2}\right\|_{\infty}\right] \\
& =\frac{\|L\|_{L^{1}} T^{\alpha}\left[\Gamma(\beta+1)+\|\ell\|_{L^{1}} T^{\beta}\right]}{\Gamma(\beta+1) \Gamma(\alpha+1)}\left\|u_{1}-u_{2}\right\|_{\infty}
\end{aligned}
$$

Hence by the assumption of the theorem we have that $\mathrm{P}$ is a contraction. Then in view of the Banach fixed point theorem, $\mathrm{P}$ has a unique fixed point which corresponds to the solution of equation (2).

\section{Set-valued problem}

We establish the existence results for the integral inclusion (1) when the right hand side has convex as well as non-convex values. The study is taken in view of fixed point theorems of set-valued functions.

Definition 17 A function $\mathfrak{u}(.) \in \mathrm{C}\left(\mathrm{J}, \mathbb{R}^{\mathrm{n}}\right)$ is said to be a solution of (1) if there exists $\mathrm{f} \in \mathrm{L}^{1}\left(\mathrm{~J}, \mathbb{R}^{\mathrm{n}}\right)$ such that $\mathrm{f} \in \mathrm{F}(\mathrm{t}, \mathrm{u}(\mathrm{t}), v(\mathrm{t}))$, where $v(\mathrm{t})=$ 
$I^{\beta} k(t, u(t))$ and

$$
u(t)=\int_{0}^{t} \frac{(t-\tau)^{\alpha-1}}{\Gamma(\alpha)} f(\tau) d \tau \quad \text { for all } t \in J
$$

Using Remark 11, we obtain the following result.

Lemma 18 Let $\mathrm{f} \in \mathrm{F}(\mathrm{t}, \mathrm{u}(\mathrm{t}), \boldsymbol{v}(\mathrm{t}))$, then $\mathrm{u}(\mathrm{t})=\mathrm{I}_{\mathfrak{a}}^{\alpha} \mathrm{f}(\mathrm{t})$ is a solution for inclusion (1).

Let us illustrate the following assumptions.

\section{Assumption $\mathrm{H} 6$}

The set-valued function $\mathrm{F}: \mathrm{J} \times \mathbb{R}^{\mathfrak{n}} \rightarrow \mathcal{P}_{\mathrm{c}, \mathrm{cp}}\left(\mathbb{R}^{\mathrm{n}}\right)$ is Carathéodory.

Assumption $\mathbf{H 7}$ Assume that

$$
\|\mathrm{F}(\mathrm{t}, \mathrm{u}, v)\| \leqslant \rho(\mathrm{t})\left(\|\mathrm{u}\|_{\infty}+\|v\|_{\infty}\right),
$$

where $\rho \in \mathrm{L}^{1}\left(\mathrm{~J}, \mathbb{R}_{+}\right)$.

Theorem 19 (Convex case) Let H3, H6 and H'7 hold. Then the integral inclusion (1) has at least one solution $\mathfrak{u}(\mathfrak{t}) \in \mathcal{B}$.

Proof: Now we show that the assumptions of Lemma 12 are satisfied. By H7 it follows that

$$
\|\mathrm{F}(\mathrm{t}, \mathrm{u}, v)\| \leqslant \rho(\mathrm{t})\left(\|\mathrm{u}\|_{\infty}+\|v\|_{\infty}\right) .
$$

But $F$ has a compact values in $\mathbb{R}$, then for every $u \in \mathcal{B}$ we have $F$ is measurable. Therefore $F(t, u, v)$ is measurable and integrably bounded where $v(t):=I^{\beta} k(t, u(t))$ and consequently the set

$$
S_{\mathrm{F}}(\mathrm{u}):=\{\mathrm{f} \in \mathcal{L}, \mathrm{f}(\mathrm{t}) \in \mathrm{F}(\mathrm{t}, \mathrm{u}(\mathrm{t}), v(\mathrm{t}))\}
$$

is closed and non-empty that for all $\mathfrak{u} \in \mathcal{B}$ (Remark 10). Define the operator $\mathrm{N}(\mathrm{u})$ on $\mathcal{B}$ by

$$
N(u):=\left\{u \in \mathcal{B}: u(t)=I^{\alpha} f\left(t, u(t), I^{\beta} k(t, u(t))\right), f \in S_{F}(u)\right\} .
$$


Our aim is to show that $\mathbf{N}(\mathfrak{u})$ satisfies the conditions of Lemma 12 and this is proven in four steps.

1. $\mathbf{N}(\mathfrak{u})$ is closed and convex. Firstly in view of Remark 10, the set $S_{F}(\mathfrak{u})$ is closed. Then consequently $N$ is closed. Now let $h_{1}(t), h_{2}(t) \in N(u)$ then there exist $f_{1}, f_{2} \in S_{F}(u)$ such that

$$
h_{j}(t)=I^{\alpha} f_{j}\left(t, u(t), I^{\beta} k(t, u(t))\right), \quad j=1,2 .
$$

Let $0 \leqslant \delta \leqslant 1$, then for each $t \in J$

$$
\begin{aligned}
& {\left[\delta h_{1}+(1-\delta) h_{2}\right](t)} \\
& =\delta I^{\alpha} f_{1}\left(t, u(t), I^{\beta} k(t, u(t))\right)+(1-\delta) I^{\alpha} f_{2}\left(t, u(t), I^{\beta} k(t, u(t))\right) \\
& =I^{\alpha} \delta f_{1}\left(t, u(t), I^{\beta} k(t, u(t))\right)+I^{\alpha}(1-\delta) f_{2}\left(t, u(t), I^{\beta} k(t, u(t))\right) \\
& =I^{\alpha}\left[\delta f_{1}\left(t, u(t), I^{\beta} k(t, u(t))\right)+(1-\delta) f_{2}\left(t, u(t), I^{\beta} k(t, u(t))\right)\right]
\end{aligned}
$$

by the convexity of $S_{F}(u)$, implies that $\left[\delta f_{1}+(1-\delta) f_{2}\right] \in S_{F}(u)$ then consequently $\left[\delta h_{1}+(1-\delta) h_{2}\right](t) \in N(u)$. Hence $N(u)$ is a convex.

2. $N(B[0, r])$ is relatively compact in $\mathcal{B}$. For $u \in B[0, r]$, then for all $h(t) \in N(u)$, we have $|h(t)| \leqslant r$. That is, $N(B[0, r])$ is bounded set. Moreover, using the properties of the fractional integral operator $\left(\mathrm{I}^{\alpha}\right)$, we have that for $0 \leqslant t_{1} \leqslant t_{2} \leqslant T$ such that $\left|t_{2}-t_{1}\right| \leqslant \delta, \delta>0$ and $u \in B[0, r]$, then for all $h(t) \in N(u)$,

$$
\begin{aligned}
& \left|h\left(t_{1}\right)-h\left(t_{2}\right)\right| \\
& =\left|I^{\alpha} f\left(t_{1}, u\left(t_{1}\right), I^{\beta} k\left(t_{1}, u\left(t_{1}\right)\right)\right)-I^{\alpha} f\left(t_{2}, u\left(t_{2}\right), I^{\beta} k\left(t_{2}, u\left(t_{2}\right)\right)\right)\right| \\
& \leqslant\left[\frac{\|f\|}{\Gamma(\alpha+1)}\right]\left|\left(t_{1}^{\alpha}-t_{2}^{\alpha}+2\left(t_{1}-t_{2}\right)^{\alpha}\right)\right| \\
& \leqslant\left[\frac{2\|f\|}{\Gamma(\alpha+1)}\right]\left|\left(t_{2}-t_{1}\right)^{\alpha}\right| \\
& \leqslant\left[\frac{2\|f\|}{\Gamma(\alpha+1)}\right] \mid\left(t_{2}-t_{1}\right)^{\alpha}
\end{aligned}
$$




$$
\leqslant \frac{2 \delta^{\alpha}\|f\|}{\Gamma(\alpha+1)}
$$

which is independent of $u$ implies that $N(B[0, r])$ is equicontinuous. An application of the Arzela-Ascoli theorem yields that $\mathrm{N}$ is a compact operator. Thus $N(B[0, r])$ is relatively compact set in $\mathcal{B}$. Hence $N$ is compact.

3. $N$ is upper semi-continuous on $\mathcal{B}$. Since $N$ is closed and $N(B[0, r])$ is relatively compact, then, in view of Remark 9 , it follows that $\mathrm{N}$ is upper semi-continuous on $\mathcal{B}$.

4. The set $A$ is not unbounded. Let $(\mathfrak{u}, \lambda) \in \mathcal{B} \times(0,1)$ such that $u \in \lambda N(\mathfrak{u})$. Therefore

$$
\begin{aligned}
|u(t)| & \leqslant \lambda I^{\alpha}\left|f\left(t, u(t), I^{\beta} k(t, u(t))\right)\right| \\
& \leqslant I^{\alpha}\left|f\left(t, u(t), I^{\beta} k(t, u(t))\right)\right| \\
& \leqslant r .
\end{aligned}
$$

It follows that $A$ is bounded set. Thus in view of Lemma $12, \mathrm{~N}$ has a fixed point which corresponds to the solution of inclusion (1).

In order to study the existence for the problem (1) in non-convex case, we introduce the following assumptions.

Assumption H8 $\mathrm{F}: \mathrm{J} \times \mathbb{R}^{\mathrm{n}} \times \mathbb{R}^{\mathrm{n}} \rightarrow \mathcal{P}_{\mathrm{cl}, \mathrm{cp}}\left(\mathbb{R}^{\mathrm{n}}\right)$, ( $\left.\mathrm{t},.\right) \mapsto \mathrm{F}(\mathrm{t}, \mathrm{u}, \boldsymbol{v})$ is measurable for each $\boldsymbol{u}, \boldsymbol{v} \in \mathbb{R}^{\mathfrak{n}}$.

Assumption H9 The set-valued function $\mathrm{F}: \mathrm{J} \times \mathbb{R}^{\mathrm{n}} \rightarrow \mathcal{P}_{\mathrm{cl}, \mathrm{cp}}\left(\mathbb{R}^{\mathrm{n}}\right)$ has a function $\mathrm{L}(\mathrm{t}) \in \mathrm{L}^{1}\left(\mathrm{~J}, \mathbb{R}^{+}\right)$such that for each $\mathrm{u}_{1}, \mathrm{u}_{2}, v_{1}, v_{2}$ on $\mathrm{J}$, we have

$$
\mathrm{H}\left(\mathrm{F}\left(\mathrm{t}, \mathrm{u}_{1}, v_{1}\right), \mathrm{F}\left(\mathrm{t}, \mathfrak{u}_{2}, v_{2}\right)\right) \leqslant \mathrm{L}(\mathrm{t})\left[\left\|\mathrm{u}_{1}-\mathrm{u}_{2}\right\|_{\infty}+\left\|v_{1}-v_{2}\right\|_{\infty}\right] .
$$

Theorem 20 (Non-convex case) Let H5, H8 and H9 hold. If (5) is satisfied, then the integral inclusion (1) has at least one solution $\mathrm{u}(\mathrm{t})$ on $\mathrm{J}$. 
Proof: In order to study the existence for problem (1), using Lemma 13 we show that $\mathbf{N}$ defined in equation (6) is a contraction set-valued mapping. The proof takes two steps.

1. $N(u) \in \mathcal{P}_{\text {cl }}(\mathbb{R})$ for each $u \in \mathcal{B}$. Let $\left\{u_{m}\right\}_{m} \geqslant 0 \in N(u)$ such that $u_{m} \rightarrow \widetilde{u}$ in $\mathcal{B}$. Then $\widetilde{u} \in \mathcal{B}$ and there exists $f_{m} \in S_{F}(u)$ such that for $t \in J$

$$
u_{m}(t)=I^{\alpha} f_{m}\left(t, u(t), I^{\beta} k(t, u(t))\right) .
$$

Using that $F$ has a compact values, we get that $f_{m}$ converges to $f$ in $L^{1}(J, \mathbb{R})$ and hence, $f \in S_{F}(u)$. Then for each $t \in J$

$$
u_{m}(t) \rightarrow \widetilde{u}(t)=I^{\alpha} f\left(t, u(t), I^{\beta} k(t, u(t))\right)
$$

So $\widetilde{u} \in N(u)$.

2. There exist $\gamma<1$ such that

$$
H\left(N\left(u_{1}\right), N\left(u_{2}\right)\right) \leqslant \gamma\left\|u_{1}-u_{2}\right\|, \quad \text { for each } u_{1}, u_{2} \in \mathcal{B} \text {. }
$$

Let $\mathfrak{u}_{1}, \mathfrak{u}_{2}, v_{1}, v_{2} \in \mathbb{R}$. Then there exists $f \in F$ such that by $H 9$, $f$ satisfies

$$
\left|f\left(t, u_{1}, v_{1}\right)-f\left(t, u_{2}, v_{2}\right)\right| \leqslant L(t)\left[\left\|u_{1}-u_{2}\right\|_{\infty}+\left\|v_{1}-v_{2}\right\|_{\infty}\right],
$$

then for $h_{1}(t) \in N\left(u_{1}\right)$ where

$$
h_{1}(t)=I^{\alpha} f\left(t, u_{1}(t), v_{1}(t)\right)=I^{\alpha} f\left(t, u_{1}(t), I^{\beta} k\left(t, u_{1}(t)\right)\right),
$$

and for $h_{2}(t) \in N\left(u_{2}\right)$ where

$$
h_{2}(t)=I^{\alpha} f\left(t, u_{2}(t), v_{2}(t)\right)=I^{\alpha} f\left(t, u_{2}(t), I^{\beta} k\left(t, u_{2}(t)\right)\right),
$$

then

$$
\begin{aligned}
& \left|h_{1}(t)-h_{2}(t)\right| \\
& \leqslant I^{\alpha}\left|f\left(t, u_{1}(t), v_{1}(t)\right)-f\left(t, u_{2}(t), v_{2}(t)\right)\right|
\end{aligned}
$$




$$
\begin{aligned}
& \leqslant \frac{\mathrm{T}^{\alpha}}{\Gamma(\alpha+1)}\|\mathrm{L}\|_{\mathrm{L}^{1}}\left[\left\|\mathrm{u}_{1}-\mathrm{u}_{2}\right\|_{\infty}+\left\|v_{1}-v_{2}\right\|_{\infty}\right] \\
& \leqslant \frac{\mathrm{T}^{\alpha}}{\Gamma(\alpha+1)}\|\mathrm{L}\|_{\mathrm{L}^{1}}\left[\left\|\mathrm{u}_{1}-\mathrm{u}_{2}\right\|_{\infty}+\frac{\mathrm{T}^{\beta}}{\Gamma(\beta+1)}\|\ell\|_{\mathrm{L}^{1}}\left\|\mathrm{u}_{1}-\mathrm{u}_{2}\right\|_{\infty}\right] \\
& \leqslant \frac{\mathrm{T}^{\alpha}}{\Gamma(\alpha+1)}\|\mathrm{L}\|_{\mathrm{L}^{1}}\left[\left\|\mathrm{u}_{1}-\mathrm{u}_{2}\right\|_{\infty}+\frac{\|\ell\|_{\mathrm{L}^{1}} \mathrm{~T}^{\beta}}{\Gamma(\beta+1)}\left\|\mathrm{u}_{1}-\mathrm{u}_{2}\right\|_{\infty}\right] \\
& \leqslant \frac{\|\mathrm{L}\|_{\mathrm{L}^{1}} \mathrm{~T}^{\alpha}\left[\Gamma(\beta+1)+\|\ell\|_{\mathrm{L}^{1}} \mathrm{~T}^{\beta}\right]}{\Gamma(\beta+1) \Gamma(\alpha+1)}\left\|\mathrm{u}_{1}-\mathrm{u}_{2}\right\|_{\infty} .
\end{aligned}
$$

Denote that

$$
\gamma:=\frac{\|\mathrm{L}\|_{\mathrm{L}^{1}} \mathrm{~T}^{\alpha}\left[\Gamma(\beta+1)+\|\ell\|_{\mathrm{L}^{1}} \mathrm{~T}^{\beta}\right]}{\Gamma(\beta+1) \Gamma(\alpha+1)} .
$$

By the assumption of the theorem, it follows that

$$
\mathrm{H}\left(\mathrm{N}\left(\mathfrak{u}_{1}\right), \mathrm{N}\left(\mathfrak{u}_{2}\right)\right) \leqslant \gamma\left\|u_{1}-\mathfrak{u}_{2}\right\|_{\infty}, \quad \text { for each } \mathfrak{u}_{1}, \mathfrak{u}_{2} \in \mathcal{B} \text {, }
$$

for sufficiently small $\mathrm{T}>0$, where $\gamma<1$. This implies that $\mathrm{N}$ is a contraction set-valued mapping. Then in view of Lemma 13, $\mathrm{N}$ has a fixed point which corresponds to a solution of inclusion (1). This completes the proof.

Example 21 Consider the problem

$$
\mathrm{D}^{\alpha} \mathrm{u}(\mathrm{t}) \in \mathrm{F}(\mathrm{t}, \mathrm{u}(\mathrm{t}), v(\mathrm{t})), \quad \mathrm{t} \in \mathrm{J}:=[0,1],
$$

where $u \in C[J, \mathbb{R}], v(t):=I^{\beta} k(t, u(t)), \alpha=\beta=0.5, k(t, u):=0.5 u(t)$ and

$$
F(t, u, v)= \begin{cases}0 & \text { if } u \in \mathbb{R} \backslash\{[0,1]\}, \\ {\left[0,0.1\left(u+I^{\beta} 0.5 u\right)\right]} & \text { if } u \in[0,1] .\end{cases}
$$

$\mathrm{F}$ is Carathéodory set-valued function. And $|\mathrm{k}(\mathrm{t}, \mathrm{u})| \leqslant 0.5|\mathrm{u}(\mathrm{t})|$ where $\mathrm{p}(\mathrm{t})=$ 0.5 then, $\|p\|=0.5$. Also $\|F\| \leqslant \rho(t) \Omega_{0}(\|u\|+\|v\|)$ where $\rho(t)=0.1$, 
then $\|\rho\|_{L^{1}}=0.1$ and $\Omega_{0} \simeq 1.28$. Therefore if $r=0.21$, then in view of Theorem 19 the problem (7) has a solution.

Indeed, we can show that $k(t, u)$ is $\ell$-Lipschitz with a Lipschitz constant $\|\ell\|_{\mathrm{L}^{1}}=0.5$. Moreover, $\mathrm{F}$ satisfies the assumption $\mathrm{H} 9$ with $\mathrm{L}(\mathrm{t})=0.1$, then $\|\mathrm{L}\|_{\mathrm{L}^{1}}=0.1$. Therefore if for $\gamma \simeq 0.17<1$, we have that $\mathrm{F}$ is a contraction set-valued mapping. Thus in view of Theorem 20, the problem (7) has a solution.

\section{Conclusion}

- We established the existence of locally solutions for diffeo-integral inclusion of Sobolev-type of fractional order because this type of differential inclusion has many applications in intermediate physical processes, convection-diffusion and transport-diffusion processes [32]. Also this type of problems involving the generalized telegraph equations of fractional order [33].

- If $\mathrm{F}(\mathrm{t}, \mathrm{u}(\mathrm{t}), \boldsymbol{v}(\mathrm{t}))$ has nonempty closed convex values in $\mathbb{R}^{\mathrm{n}}$, inclusion (1) reduces to control system where $v(t)=I^{\beta} k(t, u(t))$ is the control variable. This system is complectly controllable if for all $\mathfrak{u}_{0}, \mathfrak{u}_{1} \in \mathbb{R}^{n}$ there exists a continuous control $v(t) \in \mathbb{R}^{m}$ such that $\mathfrak{u}(0)=\mathfrak{u}_{0}$ and $\mathfrak{u}(\mathbf{T})=\mathfrak{u}_{1}[34]$. Since, see Definition 7 ,

$$
u(0)=0 \quad \text { and } \quad u(t)=I^{\alpha} f(t)=\frac{t^{\alpha-1} * f(t)}{\Gamma(\alpha)},
$$

implies

$$
u(T)=\frac{T^{\alpha-1} * f(T)}{\Gamma(\alpha)}, \quad f(T) \neq 0 .
$$

Then by arbitrary choice of $\mathfrak{u}_{0}$ and $\mathfrak{u}_{1}$ we can set $\mathfrak{u}_{0}=0$ and $\mathfrak{u}_{1}=\mathfrak{u}(T)$. Hence system (1) is controllable or completely controllable on J. 
Acknowledgment The author thanks the anonymous referee for his/her helpful suggestions for the improvement of this paper. Also many thanks to Professor Danny for his useful comments.

\section{References}

[1] R. Hilfer, Fractional diffusion based on Riemann-Liouville fractional derivatives, J. Phys. Chem. Bio. 104(2000) 3914-3917. E2

[2] R. Hilfer, The continuum limit for self-similar Laplacians and the Green function localization exponent, 1989, UCLA-Report 982051. E2

[3] B. Ross, Fractional Calculus and its Applications, Vol. 457 of Lecture Notes in Mathematics, Springer, Berlin, 1975. E2

[4] R. Hilfer, Applications of Fractional Calculus in Physics, World Scientific Pub.Co.: Singapore, 2000. E2

[5] K. S. Miller and B. Ross, An Introduction to The Fractional Calculus and Fractional Differential Equations, John-Wily and Sons, Inc., 1993. $\mathrm{E} 2, \mathrm{E} 7, \mathrm{E} 8$

[6] I. Podlubny, Fractional Differential Equations, Acad.Press, London, 1999. E2, E4, E7, E8

[7] V. Kiryakova, Generalized Fractional Calculus and Applications, Pitman Res. Notes Math. Ser., Vol. 301, Longman/Wiley, New York, 1994. E2, E7

[8] S. G. Samko, A. A. Kilbas, O. I. Marichev, Fractional Integrals and Derivatives (Theory and Applications), Gorden and Breach, New York, 1993. E2, E7

[9] K. B. Oldham and J. Spanier, The Fractional Calculus, Math. in Science and Engineering, Acad. Press, New York/London, 1974. E2, E7 
[10] A. M. A. El-Sayed, A. G. Ibrahim, Multi-valued fractional differential equations, Appl. Math. Comput. 68(1995) 15-25. E2

[11] A. G. Ibrahim, A. M. A. El-Sayed, Define integral of fractional order for set valued function, J. Frac. Calculus 11 (May 1997). E2

[12] A. M. A. El-Sayed, A. G. Ibrahim, Set valued integral equations of fractional-orders, Appl. Math. Comp. 118(2001) 113-121. E2

[13] N. S. Papageeorgion, On integral inclusion of Volterra type in Banach spaces, Czechoslovak Math. J. 42(1992) 693-714. E2

[14] N. S. Papageeorgion, On non convex valued Volterra integral inclusions in Banach spaces, Czechoslovak Math. J. 44(1994). E2

[15] S. Aizicovici, V. Staicu, Continuous selections of solutions sets to Volterra integral inclusions in Banach spaces, Elec. J. Diffe. Equa. Vol. 2006(2006) 1-11. E2

[16] M. Kanakaraj, K. Balachadran, Existence of solutions of Sobolev-type semilinear mixed integrodifferential inclusions in Banach spaces, $J$. of Applied and Stochastic Analysis 16:2(2003) 163-170. E2

[17] K. Balachandar and J. P. Dauer, Elements of Control Theory, Narosa Publishing House, 1999. E3

[18] L. V. Kantorovich and G. P. Akilov, Functional Analysis, Pergamon Press, Oxford,1982. E3

[19] K. Deimling, Nonlinear Functional Analysis, Springer-Verlag,1985. E3

[20] D. R. Smart, Fixed Point Theorems, Cambridge University Press, 1980. E3

[21] C. Avramescu, A fixed point theorem for multivalued mappings, Electronic. J. Qualitative Theory of Differential Equations. Vol. 17 (2004) 1-10. E3, E8 
[22] K. Demling, Multivalued Differential Equations, Walter de Gruyter, New York, 1992. E3, E8

[23] J. P. Aubin, A. Cellina. Differential Inclusions. Springer, Berlin, 1984. $\mathrm{E} 4$

[24] V. Barbu. Nonlinear Semigroups and Differential Equations in Banach Spaces. Noordhoff international Pupl. Leyden, 1976. E4

[25] S. Hu, N. S. Papageeorgion. Handbook of Multivalued Analysis, Vol. I: Theory. Kluwer, Dordrecht, 1997. E4, E8

[26] S. Hu, N. S. Papageeorgion. Handbook of Multivalued Analysis, Vol. II: Applications. Kluwer, Dordrecht, 2000. E4

[27] A. G. Kartsatos, K. Y. Shin. Solvability of functional evolutions via compactness methods in general Banach spaces. Nonlinear Anal., 21(1993) 517-535. E4

[28] N. H. Pavel. Nonlinear Evolution Operators and Semigroups, Lecture Notes in Mathematics, Vol. 1260. Springer, Berlin, 1987. E4

[29] I. I. Vrabie, Compactness Methods for Nonlinear Evolutions. Longman, Harlow, 1987. E4

[30] M. Kisielewicz. Differential Inclusions and Optimal Control. Dordrecht, The Netherlands, 1991. E6

[31] C. Avramescu, A fixed point theorem for multivalued mappings, Electronic. J. Qualitative Theory of Differential Equations. Vol. 17 (2004) 1-10. E8

[32] A. M. A. El-Sayed, F. M. Gaafar, Fractional calculus and some intermediate physical processes, Appl. Math. and Comp. 144(2003) 117-126. E17 
[33] R. C. Cascaval, E. C. Eckstein, C. L. Frota, and J. A. Goldstein, Fractional telegraph equations, J. Math. Anal. Appl. 276 (2002) 145-159. E17

[34] R. W. Ibrahim, Continuous solutions for fractional integral inclusion in locally convex topological space, Appl. Math. J. Chinese Univ. 24(2)(2009) 175-183. E17

\section{Author address}

1. Rabha W. Ibrahim, School of Mathematical Sciences, Faculty of Sciences and Technology, UKM, Malaysia.

mailto:rabhaibrahim@yahoo.com 